\title{
Peritonsillar Involvement in Pyoderma Gangrenosum associated with Ulcerative Colitis
}

\author{
Yu Mi Byeon, Jun Lee, Sang Jun Lee, Chol Jin Park, Na Ra Yun, Young Dae Kim, Chan Guk Park, \\ Man Woo Kim \\ Department of Internal Medicine, Chosun University College of Medicine, Gwangju, Korea
}

Peritonsillar abscess is a common deep throat infection. Early diagnosis and prompt, appropriate management of a peritonsillar abscess prevents mortality. A 45-year-old woman on steroids for an ulcerative colitis (UC) exacerbation presented with sore throat and multiple skin ulcers on her left forearm and right foot. Computed tomography of the neck revealed a peritonsillar abscess. Gram staining and culture of the abscess were negative, and a skin biopsy suggested pyoderma gangrenosum (PG). The final diagnosis was peritonsillar involvement of steroid-refractory PG-associated UC. The patient showed a complete response to infliximab. Here, we report a case of successful infliximab treatment for peritonsillar involvement of steroid-refractory PGassociated UC. (Intest Res 2014;12:153-156)

Key Words: Pyoderma gangrenosum; Ulcerative colitis; Peritonsillar abscess

\section{INTRODUCTION}

Pyoderma gangrenosum $(\mathrm{PG})$ is a rare inflammatory skin disease characterized by painful nodules or papulopustules that rapidly develop into ulcers with irregular margins. PG seldom presents as atypical nodules or aseptic abscesses. ${ }^{1,2}$ PG lesions, a cutaneous manifestation of IBD, can occur anywhere on the body, but the most common sites are the shins and regions adjacent to stomas. ${ }^{3,4}$ We report here the case of a PG lesion at an atypical site: the peritonsillar area presenting with an abscess. To our knowledge, this is the first report of PG-associated UC presenting with a peritonsillar abscess.

\section{CASE REPORT}

A 45-year-old woman with a history of UC presented with

Received September 6, 2013. Revised November 20, 2013.

Accepted January 22, 2014.

Correspondence to Jun Lee, Department of Internal Medicine, Chosun University College of Medicine, 365 Pilmun-daero, Dong-gu, Gwangju 501-

717, Korea. Tel: +82-62-220-3012,Fax: +82-62-224-5494, E-mail: leejun@ chosun.ac.kr

Financial support: None. Conflict of interest: None. bloody diarrhea and abdominal pain that worsened 10 days before visiting the hospital. Over the previous 3 years, she was treated with oral sulfasalazine and suppository mesala-

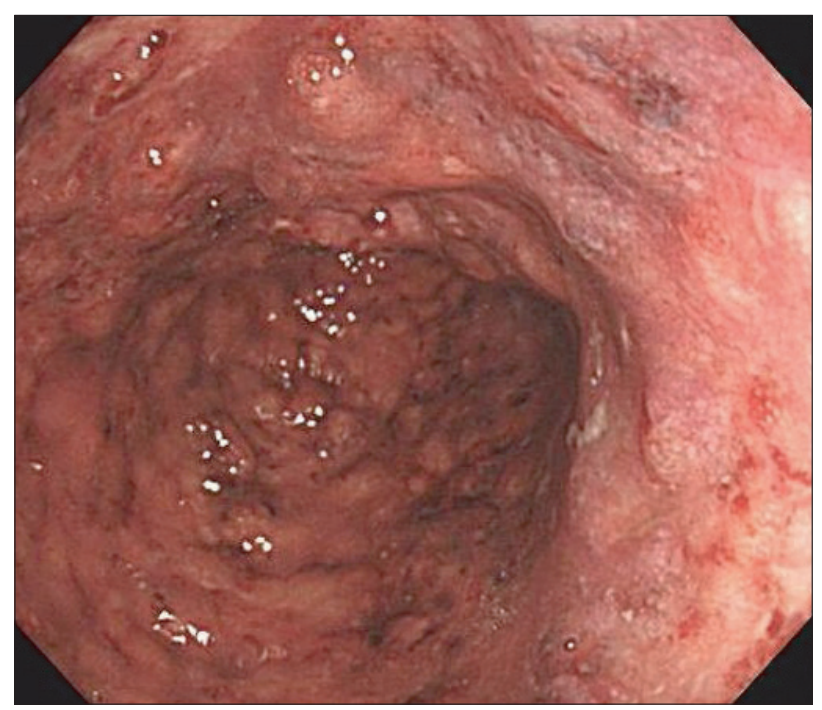

Fig. 1. Colonoscopic finding. Colonoscopy shows diffuse ulcers and nodularity with mucosal edema and hemorrhage at the sigmoid colon and rectum.

(c) Copyright 2014. Korean Association for the Study of Intestinal Diseases. All rights reserved.

This is an Open Access article distributed under the terms of the Creative Commons Attribution Non-Commercial License (http://creativecommons.org/licenses/by-nc/3.0) which permits unrestricted non-commercial use, distribution, and reproduction in any medium, provided the original work is properly cited. 
zine. Colonoscopy showed diffuse ulcers and nodules with mucosal edema and hemorrhage (Fig. 1). The patient was diagnosed with a severe episode of UC and was treated with prednisolone ( $40 \mathrm{mg} /$ day). On the seventh day of admission, her UC worsened and she had an increased number of bloody stools and a fever $\left(38.7^{\circ} \mathrm{C}\right)$. The patient also developed a sore throat and painful nodules on her left forearm and foot that progressed rapidly to ulcers with irregular margins (Fig. 2A). Biopsy of a skin lesion revealed massive lymphocytic, neutrophilic infiltration in the dermal interstitium with abscess formation (Fig. 2B). On oral examination for her sore throat, the tonsils were found to be extremely swollen and fluctuant with pus, which was collected for gram staining and culture analysis. CT of the neck revealed a hy-
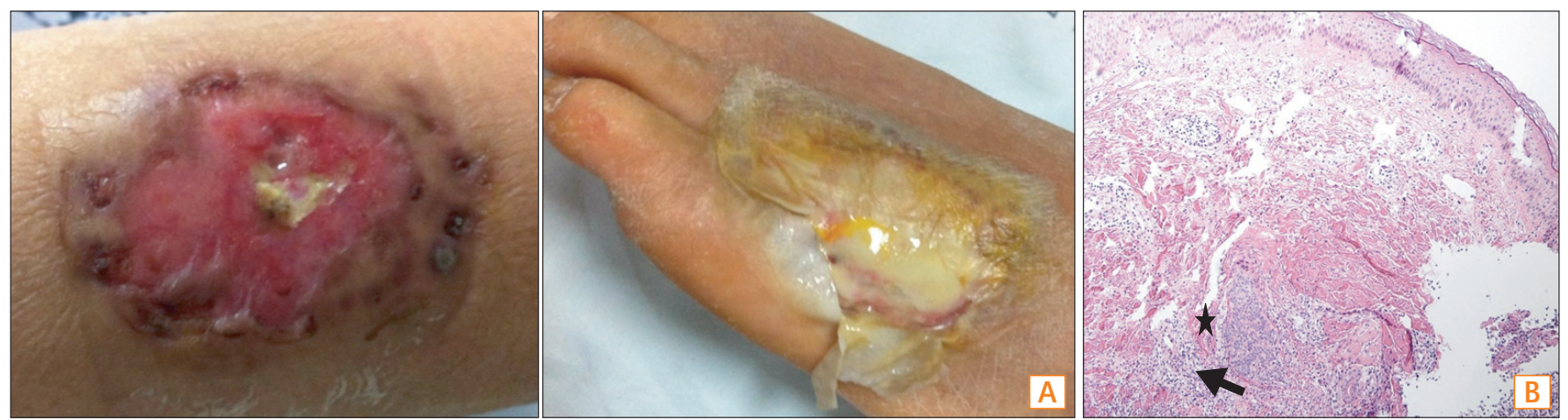

Fig. 2. Skin lesions and biopsy finding. (A) A large ulcer with edema, exudate, and pus on the left forearm and foot (B) Massive lymphocytic and neutrophilic infiltration (arrow) of the dermal interstitium (black star) suggestive of pyoderma gangrenosum (PG) (HEE stain, $\times 100)$.
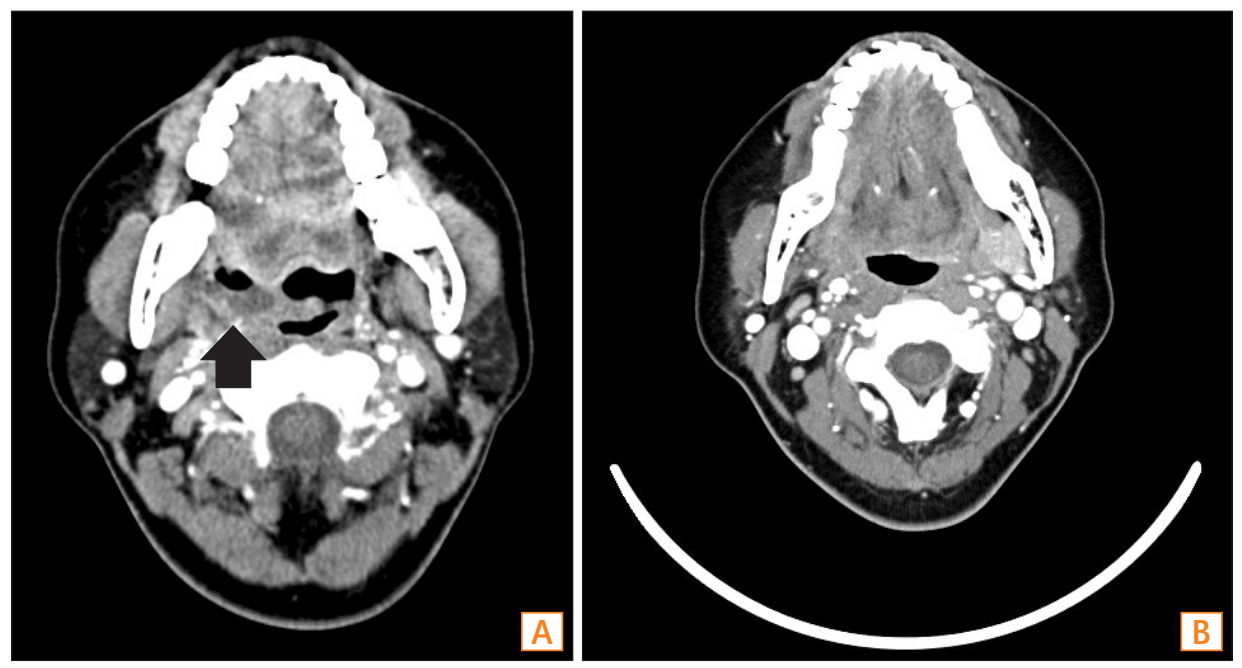

Fig. 3. Neck CT findings. (A) Computed tomography(CT) of the neck shows a hypodense mass with gas formation (arrow) suggestive of peritonsillar abscess formation. (B) A follow-up CT of the neck 3 weeks after infliximab treatment shows that the previous abscess has disappeared.
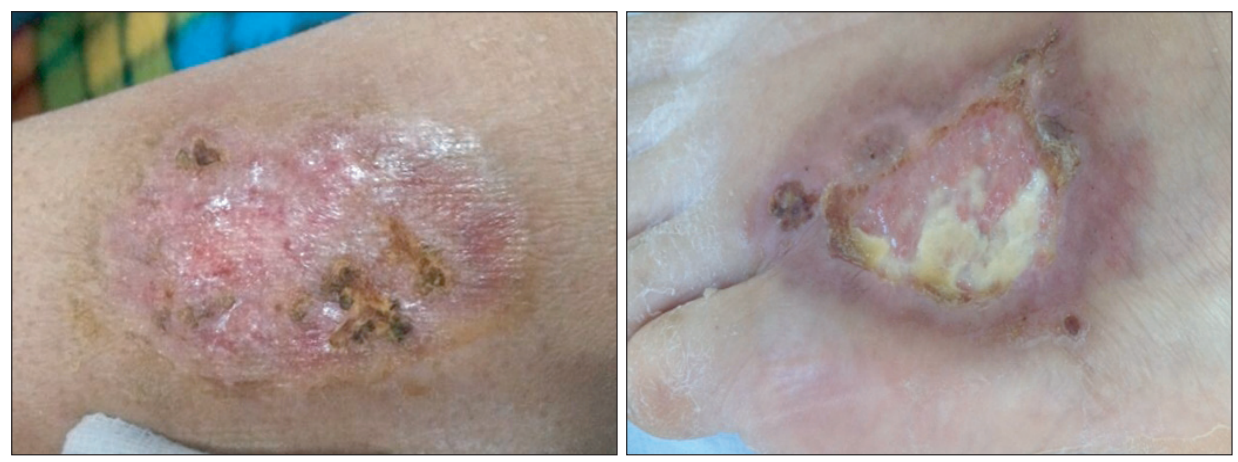

Fig. 4. Follow-up of skin lesions. Three weeks after infliximab treatment, the ulcers reduced in size and are covered with regenerated epithelium. 
podense mass with gas formation consistent with a peritonsillar abscess (Fig. 3A). The abscess was incised and drained and the patient was administered empirical intravenous antibiotics. Gram staining, and bacterial and fungal cultures were negative; therefore, antibiotics were discontinued. We diagnosed steroid-refractory PG-associated UC with peritonsillar involvement and a sterile abscess. For treatment of the steroid-refractory UC, the patient received $5 \mathrm{mg} / \mathrm{kg}$ of infliximab intravenously at 0,2 , and 6 weeks, and then every 8 weeks thereafter. The sterile peritonsillar abscess disappeared (Fig. 3B), and the skin lesions regressed significantly 4 weeks after the first infliximab infusion (Fig. 4). The patient was discharged and has been receiving infliximab as maintenance therapy.

\section{DISCUSSION}

Pyoderma gangrenosum $(\mathrm{PG})$ is a rare, immune-mediated ulcerating skin disease. The cause of PG remains unknown, but up to $50 \%$ of cases occur in association with other conditions such as IBD, connective tissue disease, or hematological disorders. ${ }^{5,6}$ PG skin lesions are commonly associated with IBD and occur in approximately $1-2 \%$ of patients with IBD. In Korea, the incidence of PG-associated IBD is unknown with few reported cases. ${ }^{7}$ Some evidence suggests that it is not related with the clinical activity of the underlying intestinal disease, but sometimes coincides with disease exacerbation or treatment failure. ${ }^{4} \mathrm{PG}$ is clinically diagnosed based on characteristic features and the exclusion of other specific skin disorders. ${ }^{8}$ The typical features of PG are single or multiple erythematous papules or pustules, but subsequent necrosis of the dermis leads to the development of deep ulcers that contain purulent material that is sterile on culture. However, PG rarely presents as atypical nodules or aseptic abscess. ${ }^{1,2}$

PG lesions can occur anywhere on the body. The most common sites are the shins and regions adjacent to stomas, but oral involvement is uncommon. Previous reports of prominent involvement of the oral mucosa in patients with PG indicate that the most common sites are the tongue, palate, lips, buccal mucosa, and gingiva. ${ }^{9}$ In this case, PG occurred on the left forearm and right foot, and in the peritonsillar space. The sterile abscess in the peritonsillar space was a variant of PG. Peritonsillar abscess is a common deep throat infection; early diagnosis and prompt, appropriate management are critical to avoid mortality. Standard treatment is antibiotics, incision, and drainage.

However, the peritonsillar lesion in this case presented as a sterile abscess, which is a variant presentation of PG-associated UC. In contrast with a pyogenic abscess, PG must be treated immediately with immunosuppressive drugs. Therefore, when a UC patient presents with a peritonsillar abscess, peritonsillar involvement of PG should be considered a differential diagnosis.

There are no uniform guidelines for the effective treatment of PG. Traditional treatment involves the use of systemic steroids and immunosuppressive drugs such as cyclosporine. ${ }^{10}$ Recently, anti-tumor necrosis factor-alpha biologic drugs have extended the therapeutic choice for IBD. In a controlled trial for $\mathrm{PG}$, a larger number of patients in the infliximabtreated group $(46 \%, 6 / 13)$ showed improvement compared with those in the placebo group $(6 \%, 1 / 17)$. Twenty of twenty-nine patients $(69 \%)$ on infliximab showed a beneficial clinical response. ${ }^{11}$ In our case, the patient developed PG while on steroid treatment. Therefore, steroid-refractory PG-associated UC was diagnosed and infliximab treatment was initiated. The patient showed good clinical response. Although more data are needed to determine the efficacy and safety of infliximab for steroid-refractory PG-associated UC, infliximab is a therapeutic option for steroid-refractory PGassociated UC.

In conclusion, PG lesions can occur anywhere on the body, but peritonsillar involvement is rare. PG rarely presents as atypical nodules or sterile abscesses. We report the case of a patient with PG who presented with a peritonsillar abscess at an atypical site.

\section{REFERENCES}

1. Johnson JL, West DA, Haggstrom AN. Pyoderma gangrenosum associated with an aseptic splenic abscess in a patient with neurofibromatosis. Pediatr Dermatol doi: 10.1111/pde.12129. Published online ahead of print 28 March 2013.

2. Allen CP, Hull J, Wilkison N, Burge SM. Pediatric pyoderma gangrenosum with splenic and pulmonary involvement. Pediatr Dermatol 2013;30:497-499.

3. Tavarela Veloso F. Review article: skin complications associated with inflammatory bowel disease. Aliment Pharmacol Ther 2004;20(Suppl 4):50-53.

4. Callen JP. Pyoderma gangrenosum. Lancet 1998;351:581-585.

5. von den Driesch P. Pyoderma gangrenosum: a report of 44 cases with follow-up. Br J Dermatol 1997;137:1000-1005.

6. Crowson AN, Mihm MC, Jr., Magro C. Pyoderma gangrenosum: a review. J Cutan Pathol 2003;30:97-107.

7. Yoo YW, Jung SH, Lee YJ, et al. A case of pyoderma gangrenosum associated with ulcerative colitis. Intest Res 2008;6:80-84. 
8. Van Assche G, Dignass A, Bokemeyer B, et al. Second European evidence-based consensus on the diagnosis and management of ulcerative colitis part 3: special situations. J Crohns Colitis 2013;7:1-33.

9. Paramkusam G, Meduri V, Gangeshetty N. Pyoderma gangrenosum with oral involvement - case report and review of the literature. Int J Oral Sci 2010;2:111-116.
10. Reichrath J, Bens G, Bonowitz A, Tilgen W. Treatment recommendations for pyoderma gangrenosum: an evidence-based review of the literature based on more than 350 patients. J Am Acad Dermatol 2005;53:273-283.

11. Brooklyn TN, Dunnill MG, Shetty A, et al. Infliximab for the treatment of pyoderma gangrenosum: a randomised, double blind, placebo controlled trial. Gut 2006;55:505-509. 